\title{
Sclérodermie et réhabilitation prothétique : à propos d'un cas
}

\section{Scleroderma and prosthetic rehabilitation: clinical report}

\section{Résumé}

MOTS-CLEFS :
- Sclérodermie,
microstomie, prothèse
complète
KEYWORDS:
- Scleroderma,
microstomia, complete
removable denture

$\operatorname{AOS} n^{\circ} 277-2016$
L'élaboration d'un plan de traitement et la décision thérapeutique associée est particulièrement délicate ò entreprendre lorsqu'un patient présente un degré d'ouverture buccale très limité (microstomie). En effet, du fait de cet obstacle, la réalisation de soins dentaires et de toute réhabilitation prothétique se révèle complexe. Cet article aborde une réhabilitation prothétique par prothèse amovible complète chez une patiente atteinte de sclérodermie et présentant une microstomie associée. Les étapes cliniques décrites prennent en compte les adaptations indispensables liées ò cette difficulté.
Abstract

\begin{abstract}
The devising of a treatment plan and the associated therapeutic decision are particularly delicate for a patient with very limited mouth opening (microstomia). Indeed, dental care and prosthetic rehabilitation can be very complex owing to this constraint. This article describes how a complete removable denture can be used for the prosthetic rehabilitation of a patient suffering from microstomia due to scleroderma. The clinical stages described here take into account the necessary adaptations that this constraint involves.
\end{abstract}

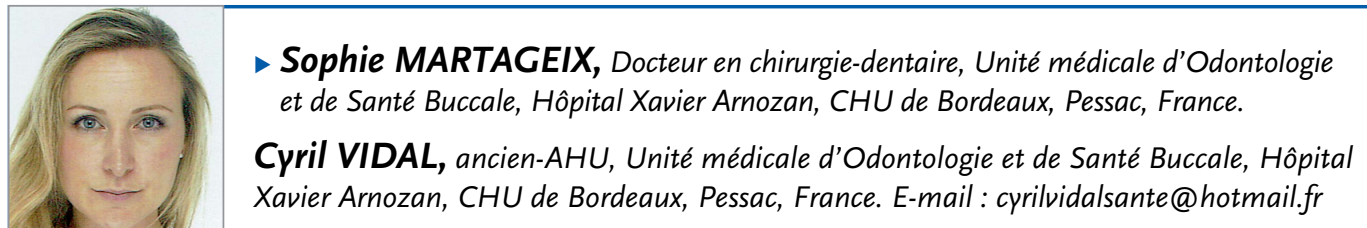

Sébastien LERICI, ancien-AHU, Unité médicale d'Odontologie et de Santé Buccale, Hôpital Xavier Arnozan, CHU de Bordeaux, Pessac, France.

Caroline BERTRAND, PU-PH, Unité médicale d'Odontologie et de Santé Buccale, Hôpital Xavier Arnozan, CHU de Bordeaux, Pessac, France.

\section{INTRODUCTION}

De nombreux obstacles sont rencontrés au sein d'un cabinet dentaire tout au long d'une carrière professionnelle. Ils peuvent être liés à l'organisation du cabinet, à la relation avec la patientèle, le prothésiste ou à des difficultés inhérentes aux soins.

Cet article expose les difficultés rencontrées lors d'une réhabilitation prothétique complète bimaxillaire chez une patiente présentant une microstomie, conséquence d'une maladie auto-immune rare dont la patiente est atteinte, la sclérodermie.
La sclérodermie est une maladie auto-immune d'étiologie encore mal connue. Elle fait partie des connectivites, un groupe de maladies internes qui ont en commun une atteinte diffuse, inflammatoire et chronique du tissu conjonctif. Le tissu conjonctif est indispensable au bon fonctionnement des organes. Cette maladie touche principalement la peau, et les organes internes dans ses formes diffuses. Le dépôt anormalement abondant de collagène de types I et III, donne lieu à une fibrose tissulaire progressive, probablement en raison des fibroblastes immunologiquement suractivés. Ces dépôts se traduisent par un 
épaississement et un durcissement cutanés. La peau perd son élasticité. Les effets les plus spectaculaires de la maladie portent sur ces changements cutanés mais aussi sur des altérations vasculaires. Les petites artères et artérioles sont affectées entraînant un épaississement de leur paroi et une réduction du diamètre de la lumière. Cette dysfonction vasculaire est l'une des premières altérations détectées et constitue l'élément initiateur dans les différentes formes de la maladie (1).

\section{CLASSIFICATION DES SCLÉRODERMIES}

La sclérodermie peut revêtir un large éventail de formes cliniques. Sa classification actuelle est basée sur l'ampleur et les caractéristiques de la sclérose de la peau et sur le niveau d'atteinte des organes (2).

Ainsi, il existe deux types de sclérodermie :

$\checkmark$ la sclérodermie localisée

I la sclérose systémique progressive, elle-même subdivisée en deux formes cliniques (3):

- Sclérose systémique limitée ou syndrome de CREST.

- Sclérose systémique diffuse.

Dans la sclérodermie systémique progressive, en plus de la peau, le cœur, les voies aérodigestives et les reins peuvent être atteints (4). Le pronostic vital peut être engagé $(5,6)$.

Le syndrome de CREST est une forme limitée de la sclérose systémique progressive, regroupant cinq symptômes majeurs à l'origine de son nom (Cutaneous calcifiation, Raynaud's phenomenon, Esophageal motility disorders, Sclerodactyly, Telangiectasia) (7). La présence de trois de ces cinq symptômes permet de diagnostiquer la maladie : des calcinoses cutanées, un syndrome de Raynaud, des troubles moteurs oesophagiens, une sclérodactylie, des télangiectasies (8).

\section{MANIFESTATIONS OROFACIALES}

Certaines manifestations orofaciales sont décrites au cours de la sclérodermie systémique $(\mathrm{ScS})$ mais leur prévalence est mal évaluée car peu d'études sont disponibles dans la littérature sur ce sujet. Elles peuvent être présentes dès les premiers temps de la maladie ou apparaître après de nombreuses années d'évolution (9).

Les lésions tégumentaires de la face sont souvent l'occasion d'évoquer cliniquement le diagnostic de $\mathrm{ScS}$, dans ses formes limitées ou diffuses. Elles se manifestent par une fibrose, la peau devenant scléreuse, dure, adhérente aux plans profonds, occasionnant un amincissement voire un effacement des lèvres, une perte des plis du front, des rides, une perte de la mimique donnant au visage l'apparence d'un masque (9). Les ailes nasales peuvent être atrophiées. La sclérose et l'atrophie des lèvres et des tissus péribuccaux produisent des stries péri-orales (10).

L'orifice buccal se trouve rétréci avec une limitation de l'ouverture buccale (LOB) (6). La LOB est appelée microstomie quand la distance inter labiale est inférieure à $45 \mathrm{~mm}$ ou que la distance inter incisive est inférieure à $40 \mathrm{~mm}$. Elle est considérée comme sévère si la distance inter incisive est inférieure à $30 \mathrm{~mm}$ (11). Les télangiectasies de la face ou de la muqueuse buccale sont également fréquentes et témoignent de l'atteinte micro vasculaire. En pratique, elles se manifestent par la présence de plaques rouges diffuses (Fig. 1).

Des lésions ostéo-articulaires des os de la face ont été aussi décrites (9) sous forme d'ostéolyse mandibulaire, dont la pathogénie et l'évolution sont incertaines. Il peut y avoir aussi des douleurs au niveau des articulations temporo mandibulaires (ATM) et une névralgie du nerf trijumeau $(12,9)$.

Une xérostomie est présente chez $70 \%$ de ces patients, secondaire à la fibrose des glandes salivaires. Associée à une atteinte sévère de l'œsophage, elle peut être aggravée (10). Cette xérostomie favorise une augmentation de la plaque dentaire avec majoration du risque carieux et des lésions parodontales (10). Une xérophtalmie est souvent associée (2).

Si la sclérose cutanée est au premier plan, la sclérose muqueuse doit être aussi recherchée minutieusement. Celle-ci peut entraîner un épaississement du frein lingual, l'apparition de brides vestibulaires ou une disparition des reliefs palatins. (9)

Les manifestations orofaciales de la ScS sont fréquentes et invalidantes. Ces symptômes sont vécus comme pénibles (9) avec un retentissement important sur la qualité de vie (11).

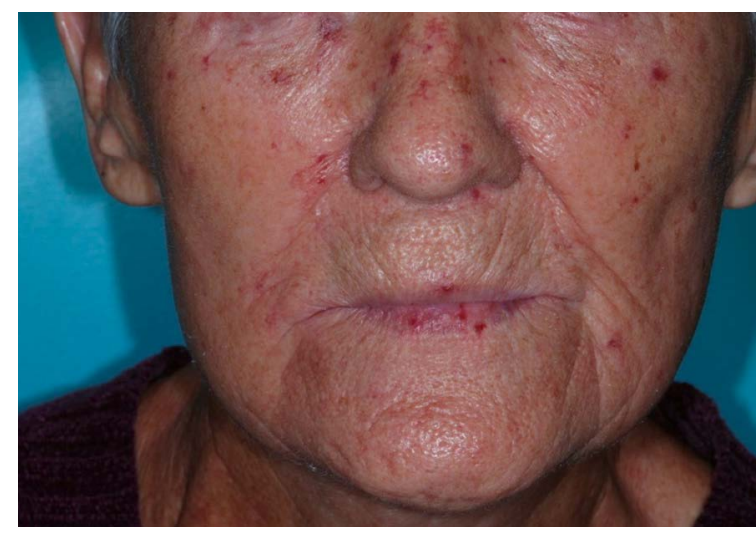

$\triangle$ Fig. 1 : Télangiectasies. 


\section{IMMUNOPATHOLOGIE}

Bien que les mécanismes responsables de cette pathologie ne soient pas encore bien élucidés, des anomalies immunologiques ont été suggérées comme jouant un rôle clé dans la pathogénèse de la maladie (13).

Les principaux acteurs cellulaires impliqués dans la physio-pathogénie de la ScS sont les cellules endothéliales, les lymphocytes T (LT) et les fibroblastes (6). Les hypothèses actuelles se focalisent sur des interactions entre des anomalies de la tolérance immunitaire, des lésions de l'endothélium de la microcirculation et une activation des fibroblastes avec synthèse excessive de la matrice extracellulaire (6).

Au plan immunopathologique, des travaux récents ont mis en relief d'une part le rôle de certaines cytokines telles que TGF- (transforming growth factor) et le PDGF (platelet derived growth factor) et d'autre part celui des lymphocytes $B(L B)$ avec la découverte d'auto-anticorps (autoAc) potentiellement pathogéniques (14). On estime à l'heure actuelle que la dysfonction endothéliale est un événement précoce, initiateur de la maladie. Les patients présentent des manifestations vasculaires très précocement sous la forme $\mathrm{du}$ syndrome de Raynaud (quasi-constant) précédant les premiers symptômes sclérodermiques de plusieurs mois voire de plusieurs années (14). Les auto-anticorps relevés dans la ScS, spécifiques des différentes formes immuno-cliniques de la maladie, constituent des outils de diagnostic et de pronostic (15).

\section{ÉVOLUTION ET PRONOSTIC}

Si la sclérodermie reste, dans la majorité des cas, limitée à la peau et aux tissus sous-cutanés (16), avec une évolution lente, en revanche, l'évolution et le pronostic de la sclérose systémique sont très variables, avec parfois une certaine gravité des atteintes viscérales (17). La survie à 10 ans est proche de $90 \%$. (6)

\section{TRAITEMENT}

La sclérodermie reste une maladie pour laquelle il n'existe pas encore de traitement efficace. La plupart des traitements disponibles, considérés comme suspensifs, permettent de traiter les symptômes et d'éviter l'évolution des manifestations cutanées ou viscérales de la maladie (6).

\section{CAS CLINIQUE}

Madame X., âgée de 78 ans, présente une sclérodermie systémique limitée à type de "CREST Syndrom » associée à une hypertension artérielle, une hypothyroïdie et une sécheresse buccale.

À l'examen clinique exobuccal, le visage de forme ovale est relativement symétrique. Le profil est concave. Des tâches rouges sont présentes sur l'ensemble du visage et au toucher, la peau manque d'élasticité. Les lèvres sont très amincies et l'ouverture buccale limitée à $35 \mathrm{~mm}$. La largeur de la cavité buccale mesurée d'une commissure à l'autre est de $40 \mathrm{~mm}$ (Fig. 2a et 2b).

À l'examen endobuccal, les arcades ont une forme ovoïde, une voûte palatine profonde en « $\mathrm{U}$ » et la hauteur des crêtes maxillaires est favorable contrairement à la crête mandibulaire qui est très résorbée. Tous ces éléments sont favorables pour assurer la sustentation, la stabilisation et la rétention d'une future prothèse. On note la présence de dents résiduelles cariées, délabrées, douloureuses et pour certaines, en dehors du plan d'occlusion (Fig. 3).

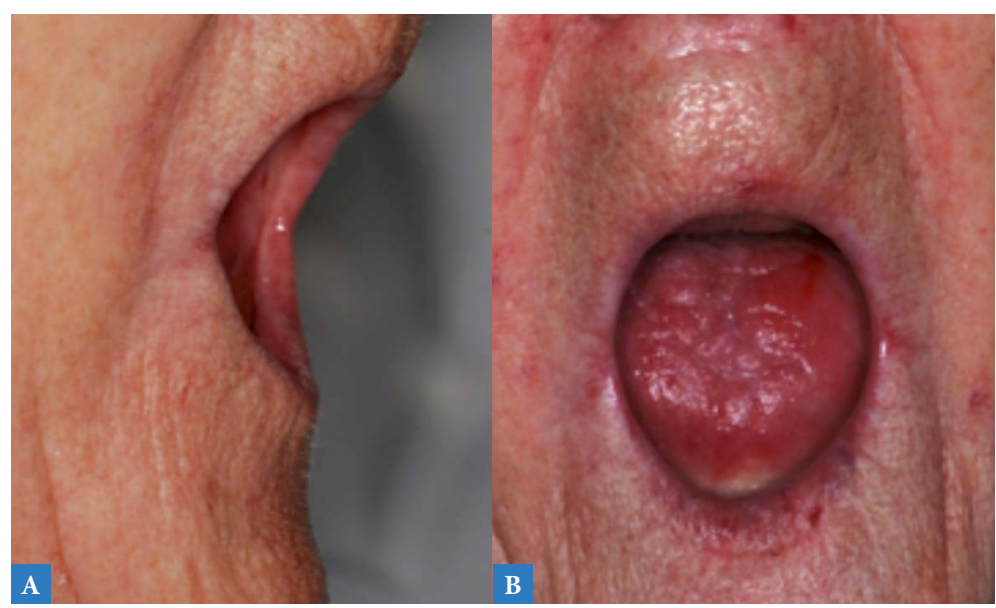

$\triangle$ Fig. 2a et $2 \mathrm{~b}$ : Limitation de l'ouverture buccale.

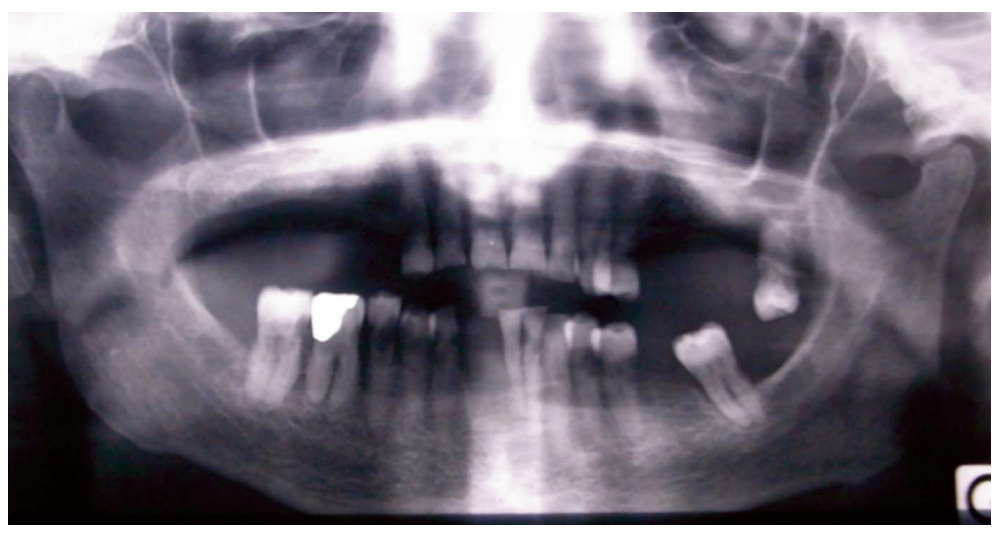

$\triangle$ Fig. 3 : Orthopantomogramme. 
Décision est prise, en accord avec la patiente, de procéder à l'extraction des dents restantes puis à une réhabilitation prothétique par prothèse amovible complète. Les obstacles cliniques à la réalisation des extractions d'abord, puis des prothèses complètes sont importants en raison de la microstomie, de la xérostomie, et des lèvres fines difficiles à étirer. Chaque étape clinique demande une réflexion et une adaptation du geste technique. Un premier jeu de prothèses complètes provisoires est réalisé pour appréhender l'ampleur des difficultés prothétiques posées par cette situation. À l'issue de la phase de cicatrisation, des prothèses complètes d'usage seront réalisées.

\section{PREMIÈRE PHASE, RÉALISATION DE PROTHÈSES AMOVIBLES COMPLÈTES TRANSITOIRES IMMÉDIATES}

Pour cette première phase, décision est prise d'augmenter le nombre de séances afin d'en raccourcir la durée, la patiente étant très vite fatiguée. Les dents postérieures maxillaires et mandibulaires sont extraites dans un premier temps jusqu'aux secondes prémolaires permettant ainsi de conserver la dimension verticale d'occlusion de la patiente intacte et d'obtenir un édentement de classe I de Kennedy. Plusieurs séances sont programmées, ces avulsions demandent beaucoup de patience tant pour la patiente que pour le praticien, l'encombrement des instruments associé à la faible ouverture buccale ne facilitant ni l'accès ni la vision du champ opératoire. De nombreuses pauses sont nécessaires au cours de chaque séance de chirurgie.

Après trois semaines de cicatrisation, les empreintes primaires sont réalisées. En raison de la présence d'une asialie et d'une ouverture buccale extrêmement limitée, le choix du matériau à empreinte s'est porté naturellement sur un alginate de classe A. Les plus petits porte-empreintes du commerce Schreinemakers ${ }^{\circ}$ à notre disposition, de taille 0 , se révèlent encore trop larges pour une insertion aisée. La question de réaliser l'empreinte au silicone de haute viscosité par pression digitale s'est posée, mais elle n'aurait pas permis de s'affranchir de la présence d'un porte-empreinte pour un soutien efficace du matériau. Au cours de cette empreinte, le geste le moins douloureux pour la patiente consiste à lui vaseliner les commissures des lèvres avant de procéder à l'insertion oblique du porte-empreinte. La finesse des lèvres donne la désagréable sensation au praticien que les lèvres pourraient se déchirer et la patiente redoute considérablement cette étape. Cette procédure nous a permis d'obtenir des empreintes de qualité acceptable et exploitables au laboratoire pour réaliser deux porte-empreintes individuels (PEI). Les empreintes secondaires anatomofonctionnelles sont alors envisagées. Les PEI se révèlent encore trop volumineux en raison de la présence des bourrelets de préhension. L'enregistrement du joint périphérique n'a pas été réalisé à la pâte de Kerr verte ${ }^{\circ}$ habituellement utilisée dans les techniques d'empreinte conventionnelles. Cet enregistrement sectoriel nécessite des insertions et désinsertions répétées, déconseillées dans le cas présent. Nous avons donc opté pour une technique d'empreinte globale avec un matériau de type polysulfure. Même si cela n'est pas conventionnel, le joint périphérique a été enregistré en même temps que la surface d'appui. Un polysulfure de haute viscosité est déposé sur les bords prothétiques et un polysulfure de moyenne viscosité dans l'intrados du PEI. Un polyéther aurait pu être préféré, cependant la présence d'embrasures cervicales marquées nous en a dissuadé. Le choix de l'utilisation des polysulfures a été décidé pour deux raisons essentielles : une viscosité qui varie peu dans les premiers instants et un temps de prise plus long contrairement au polyéther dont la viscosité évolue plus rapidement et le temps de prise est un peu court. L'insertion du PEI étant lente et délicate, ce critère était important à prendre en considération. Enfin, l'utilisation d'un matériau hydrophobe est facilitée par l'absence de salive.

Des bases d'occlusion surmontées de bourrelets en cire sont demandées. Les dents restantes ne perturbant pas le plan d'occlusion, la dimension verticale demeurant intacte, il nous a été possible de réaliser facilement l'enregistrement de la relation intermaxillaire en évitant des insertions - désinsertions répétées.

L'essayage fonctionnel est validé, les dents antérieures sont alors meulées sur le plâtre, le montage des dents est finalisé au laboratoire et les prothèses polymérisées. Les dents antérieures maxillaires sont extraites et la prothèse complète transitoire insérée en bouche.

Les conseils d'usage sont donnés à la patiente et un substitut salivaire lui est prescrit pour accroître son confort prothétique et prévenir les blessures qui pourraient être issues de la fragilité et de la sécheresse des muqueuses.

À la séance suivante, les dents antérieures mandibulaires sont extraites et la prothèse mandibulaire insérée en bouche. Les conseils d'usage sont renouvelés.

La patiente a finalement relativement bien supporté le déroulement de toutes ces séances, ses appréhensions ont été levées et l'intégration des prothèses s'en est trouvée facilitée. L'équilibration occlusale a été affinée aux cours des séances suivantes. 
DEUXIÈME PHASE, RÉALISATION DE PROTHĖSES AMOVIBLES COMPLÈTES

Après 4 mois de cicatrisation le remodelage osseux a rendu les prothèses transitoires inconfortables et moins rétentives. La patiente a eu recours, pendant plusieurs semaines, à une crème adhésive.

Durant cette seconde étape, une certaine appréhension de la patiente est réapparue face à l'idée de devoir subir de nouveau toutes ces étapes prothétiques, cependant la confiance acquise envers le praticien et la satisfaction du résultat esthétique et fonctionnel a permis de dépasser ces appréhensions.

Le choix de porte-empreintes perforés pour édenté complet de taille 0 et d'un alginate de $\mathrm{Cl} \mathrm{A}$ a été décidé. Le geste technique d'insertion a été facilité du fait de l'absence de dents résiduelles. Il a été demandé au laboratoire de prothèse de réaliser des PEI surmontés de bourrelets de préhension les plus étroits possibles (Fig. 4).

Les empreintes secondaires ont été réalisées en deux temps avec le même matériau de type polysulfure: d'abord l'enregistrement du joint périphérique avec un polysulfure de haute viscosité (Fig. 5), puis l'enregistrement de la surface d'appui avec un polysulfure de moyenne viscosité (Fig. 6a et 6b). Les deux empreintes ont même pu être réalisées en une seule séance.

Du fait de l'absence de calage dento-dentaire, il n'était plus concevable d'envisager l'enregistrement de la relation intermaxillaire avec des bases et des bourrelets en cire. Les bases d'occlusion ont donc été réalisées sur les modèles issus des empreintes secondaires avec des bourrelets en cire $\mathrm{Moyco}^{\circ}$ (Fig. 7).

Une résorption et un remodelage osseux se produisent inévitablement suite à des extractions dentaires. À la mandibule, une résorption centrifuge plus rapide et plus marquée a été décrite chez les patients sclérodermiques par Mouton et coll. (12). Ce phénomène de résorption très rapide a été observé chez notre patiente. Ceci a rendu l'enregistrement particulièrement difficile car la patiente avait tendance du fait de ces résorptions exagérées à se retrouver proche d'une classe III squelettique (Fig. 8a et 8b).

Nous avons essayé de nous rapprocher d'un bout à bout incisif lors du montage des dents. Au maxillaire, les dents prothétiques plus vestibulées que celles de la prothèse transitoire, modifient légèrement le soutien de la lèvre et du fait de la finesse des tissus, le sourire est plus difficile à accomplir par la patiente donnant l'impression d'une lèvre sous tension. Après insertion

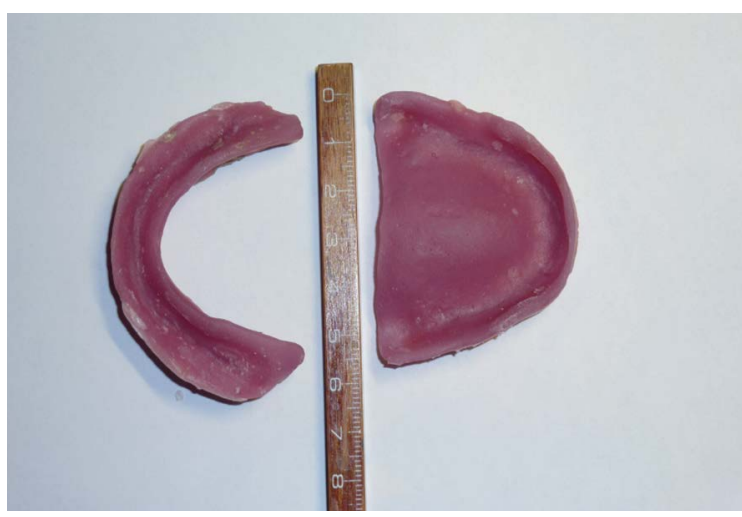

$\triangle$ Fig. 4 : PEI.

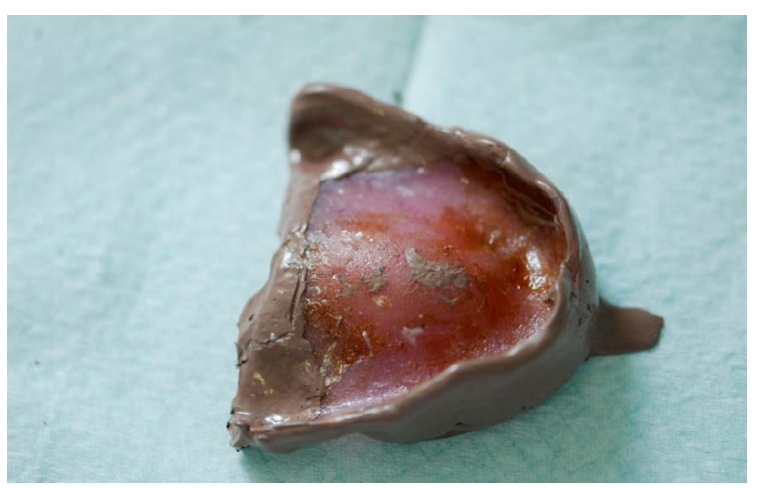

$\triangle$ Fig. 5 : Marginage.

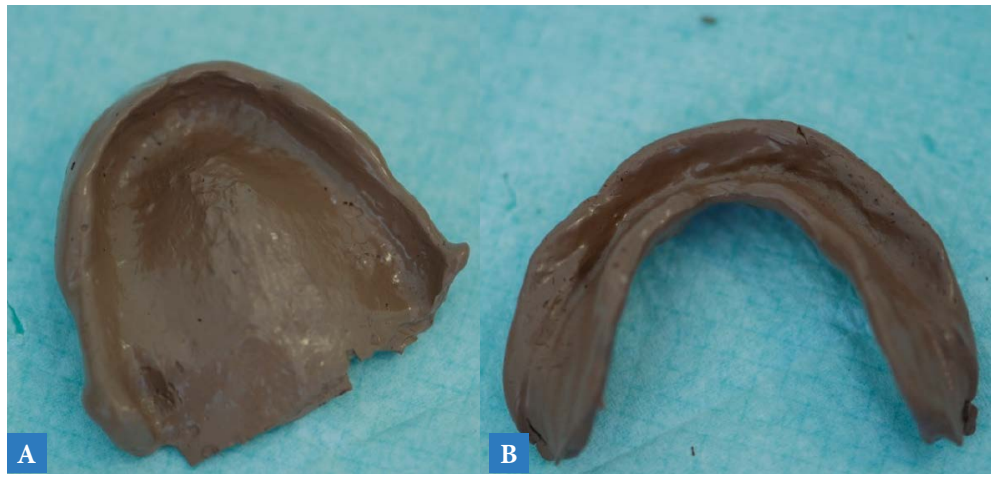

$\triangle$ Fig. 6a et 6b : Empreintes secondaires.

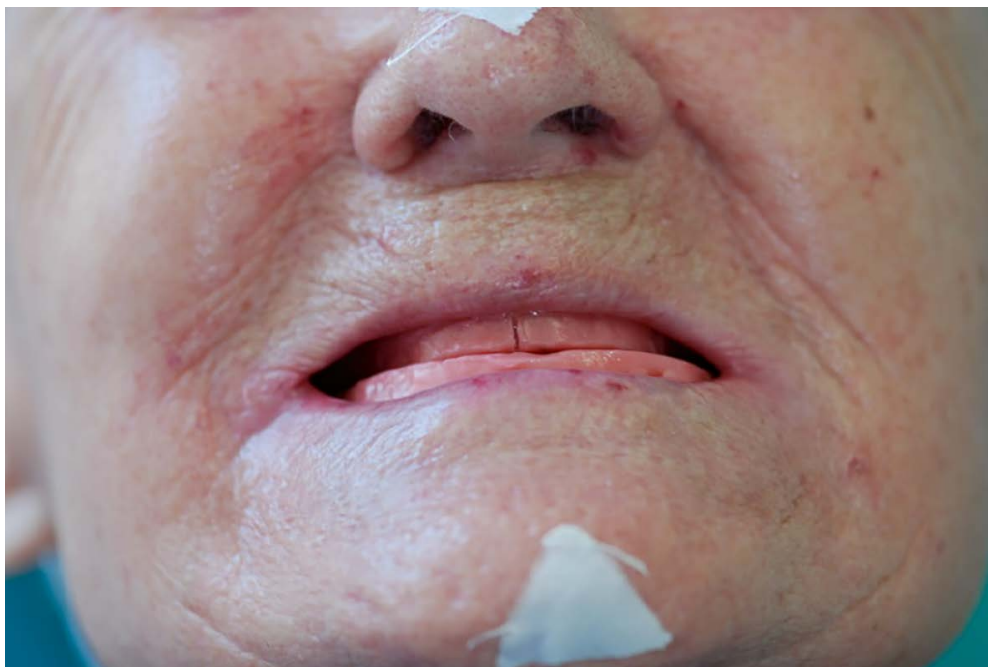

$\triangle$ Fig. 7 : Enregistrement de la dimension verticale d'occlusion. 


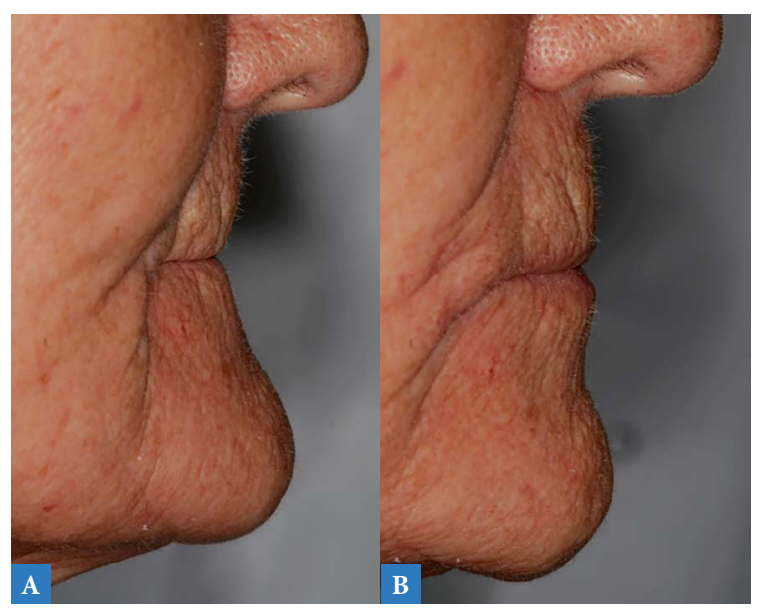

$\triangle$ Fig. 8a et $8 \mathrm{~b}$ : Modification esthétique du profil.

prothétique, la patiente très satisfaite du résultat esthétique a pu, après une semaine de port des prothèses, se détendre et s'adapter à cette nouvelle prothèse maxillaire: "Je ne la sens absolument pas, c'est comme si je ne portais pas de prothèses ».

L'insertion de la nouvelle prothèse mandibulaire a été plus délicate et plus difficile en raison de la très forte résorption qui s'est produite après les extractions. Il en a résulté un défaut de rétention et la nécessité de recourir systématiquement à l'usage d'un adhésif. Le recours à la pose de deux mini-implants symphysaires en complément de rétention a été proposé. Cette solution n'a cependant pas été retenue par la patiente. Par la suite, la patiente a constaté une très nette amélioration de sa production salivaire depuis qu'elle porte ses prothèses amovibles et un plus grand confort masticatoire dans le temps (Fig. 9).

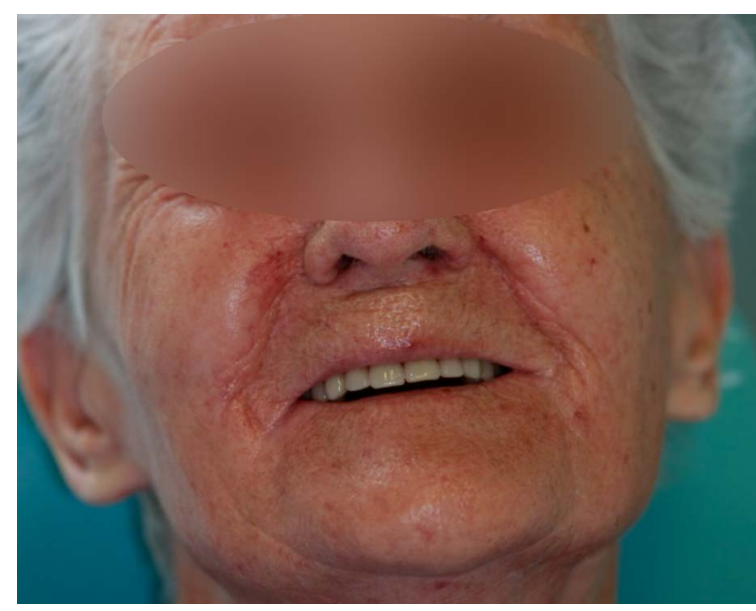

$\triangle$ Fig. 9 : Patiente le jour de la remise des prothèses.

\section{CONCLUSION}

À travers ce travail, nous avons voulu partager notre approche et démontrer, comme d'autres auteurs (18, 19, 20, 21, 22), que la microstomie, appréhendée par les praticiens, n'empêche pas d'envisager une réhabilitation prothétique qu'elle soit partielle ou complète. Dans la littérature scientifique, différentes approches, plus originales les unes que les autres, sont décrites $(18,19,20,21,22)$. Il faut savoir laisser libre cours à son imagination tout en respectant les fondamentaux de la prothèse complète. Cela permettra aux patients atteints de sclérodermie, comme tout un chacun, de bénéficier de soins dentaires et de réhabilitations prothétiques susceptibles de les aider à mieux vivre leur handicap en leur permettant de poursuivre une vie sociale et affective de meilleure qualité. 


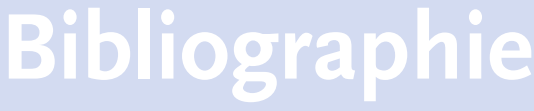

[1] Sandor G et al. Small mouths big problems? A review of scleroderma and its oral health implications. Clinical Practic 2007 ; 73(9): 831-836.

[2] Lauritano D et al. Scleroderma and CREST syndrome : a case report in dentistry. Minerva Stomatol 2011; 60(9): 443-465.

[3] Dedic A. et al. Scleroderma - from the aspect of dental medecine : case report. Medicinski Archiv 2011; 65(6): 368-370.

[4] Habif TP et al. Maladies cutanées: diagnostic et traitement. Elsevier édit, Issy-lesMoulineaux, 2008.

[5] Launay D et al. Atteinte cardiaque de la sclérodermie systémique. Revue MT Cardio 2006; 2(3): 339-354.

[6] Bessis D et al. Manifestations dermatologiques des connectivites, vasculites et affections systémiques apparentées. Vol 1 , Springer édit, Paris, 2007.

[7] Akiyama $\mathrm{Y}$ et al. Clinical, serological and genetic study in patients with CREST syndrome. Internal Medecine 2000; 39(6): 451-456.

[8] Adnan ZA et al. Diagnosis and treatments of scleroderma". Acta Medica Indonesiana 2008; 40(2): 109-112.
[9] Vincent C et al. Les manifestations buccofaciales de la sclérodermie systémique : étude de 30 patients consécutifs ». Revue de Stomatologie et de Chirurgie Maxillo-Faciale, 2010; 111(3): 128-134.

[10] Atlantar A et al. Recommandations for the care of oral involvment in patients with systemic sclerosis. American College of Rheumatology 2011; 63(8): 1126-1133

[11] Yuen HK et al. Effect of orofacial exercises on oral operture in adults with systemic sclerosis. Disability and Rehabilitation 2012; 34(1): 84-89.

[12] Mouton $\mathrm{L}$ et al. La résorption mandibulaire, une manifestation méconnue de la sclérodermie systémique. La Presse Médicale 2006; 35(4): 611-614.

[13] Ingegnoli F et al. Distinct immune profiles characterize patients with diffuse or limited systemic sclerosis. Clinical Immunology 2003; 108(1): 21-28.

[14] Martin T. Immunopathologie de la sclérodermie systémique. Médecine Thérapeutique 2008; 14(3): 149-155.

[15] Admou B et al. Autoanticorps au cours de la sclérodermie systémique : intérêt clinique et approche diagnostique. Annales de Biologie Clinique 2009; 67(3): 273-281.
[16] Toledano C et al. Localized scleroderma : a serie of 52 patients". European J Inter Med 2009; 20(3): 331-336.

[17] Haute Autorité de Santé. Sclérodermie Systémique : protocole national de diagnostic et de soins. Juillet 2008 .

[18] Bachhav VC, Aras MA. A simple method for fabrication custom selectional impression trays for making definitive impressions in patients with microstomia. European $J$ Dentistry 2012; 6(3): 244-247.

[19] Yenisey M et al. A prosthodontic management alternative for scleroderma patient. J Oral Rehabil 2005; 32(9): 696-700.

[20] Watanabe I et al. Applications of cast magnetic attachments to sectional complete dentures for a patient with microstomia : a clinical report. J Prosth Dent 2002; 88(6): 573577.

[21] Roberto B et al. Prosthetic rehabilitation for a patient with microstomia : a clinical report. J Prosth Dent 2004; 92(4): 322-327.

[22] Egan JG, Swindells SA. Novel prosthodontic alternative for patients who are edentulous and have microstomia : a case report. Special Care Dentistry 2012; 32(4): 160-164. 